\title{
DISCUSSIONS AND CRITICISM
}

\author{
Green Saharas, Grey Markets: Commercial Exploitation \\ of North African Prehistory, an Overview
}

by Paul M. Barford ${ }^{a}$

In a number of countries, the collecting of archaeological artefacts is regarded as a valid manner of public engagement with the past and has experienced an explosive growth in the last fifty years. This is due to two factors: the spread in the I970s of the use of metal detectors for hobby artefact hunting, and then in the mid I990s internet trading changed the face of the antiquities market and placed the commerce in archaeological artefacts at the reach of everybody. This in turn is currently deeply affecting public perceptions of archaeology in those countries. Thousands of people, in Europe and North America in particular, engage in collecting either through artefact hunting on local sites, while others acquire objects through purchase, driving a growing international antiquities market. This paper attempts to explore some of the wider material consequences of this general phenomenon, focusing on the collection of and commerce in prehistoric lithic material from the Sahara region. Part of it is framed around a detailed search in 2019 of the major internet portals handling this type of material.

Popular interest in the indigenous cultures of the Sahara was a legacy of European colonialism and a growing interest (from about the mid 1950s) in western societies in owning and collecting "ethnic" and "tribal art" (Graburn ed. 1977: 315; Corbey 2000) associated with an idyllic sentimentalist vision of the bon sauvage living in harmony with nature. Imaginations were fired in the 1950s and 1960s by the discoveries and popular publications of Henri Lhote (e.g., 1958) about the prehistory of the desert regions of North Africa. The enigmatic rock art he described appealed to modern aesthetics as well as New Agers; the whole issue of a primordial "Green Sahara" raised questions that resonated with environmentalists. These factors encouraged the growth of a market for collectables from this region.

In the I960s to 1980s, trade in antiquities and ethnographic objects was in the hands of knowledgeable and experienced specialist dealers with brick-and-mortar "galleries", high overheads, and limited clientele (e.g., Ede 1976). This dictated the quality, nature and cost 
3I2 $\mid$ Discussions and criticism

of the pieces offered. The market changed dramatically in 1995 with the rise of widespread internet trading (Bruhns 2000; Vitelli 2000: Chippindale and Gill 200I; Lidington 2002; Brodie 20I5; 20I7; Barker 20I8). After that watershed event, anybody with access to a computer could sell antiquities with access to a truly global market of suppliers and buyers. A significant effect of this has been a downward shift in the types of artefacts that were offered, the market shifting from limited movement of articles of relatively high value to bulk commerce in smaller items (marketed as "pieces of the past in your hand") of much less value, often a few dollars apiece. But the quantities of artefacts on the market increased, and along with it the pace of the destruction of the archaeological record to obtain them, leading to concern that this threatens the discipline itself (Brodie and Tubb 2002).

The high demand and limited licit sources of supply means that a lot of antiquities on the market today are fake or illicitly trafficked from source countries whose legislation makes archaeological and ethnographic material the property of the state and curbs its private ownership or export (Jury 2005; van Ham et al., 20II). The I970 UNESCO Convention art. 3 defines trafficked and smuggled items of cultural property as illicit (UNESCO 1970). The internal national legislation of the market countries where they are bought and sold often do not adequately regulate trade in such material, resulting in the commerce in portable antiquities often taking the form of an ambiguous grey market (Mackenzie and Yates 2016).

\section{SOURCES OF ARTEFACTS}

It is not easy to determine how Saharan Neolithic material is reaching the market. The antiquities market is very secretive, and rarely willing to share information about the dealer's sources. This is allegedly due to the need to hide this information from the competition, the truth is that it can also hide dishonest dealing practices, such as price-hiking and dubious sources of supply.

Much of the material reaching collectors through today's antiquities market is currently being obtained by the ongoing collection-driven exploitation of the archaeological record in the source countries. Some of the bulk material sold online seems to have been coming out of Marocco, while some sellers assert their material is coming from Algeria. Mali and Mauretania are also explicitly mentioned as source countries. In the majority of cases however (thousands of items), there is no location of source offered that is more precise than "Africa", "Sahara" or "deserts of Northwest Africa".

Although they usually have not secured any paperwork documenting the assertion, most dealers claim that the material they sell comes from old collections created before the 1970 UNESCO definition. This however ignores several objective facts. Much of the sort of material now regarded as collectable would neither have been available, nor considered collectable, before the changes in the antiquities market after the mid 
1990s. The number of collectors in the I970s (when the global population was 3.7 billion) would have been much smaller than in the 2020 (population 7.8 billion), and collecting was an elite activity, so there would be fewer former collections to satisfy modern demand. Also many of the artefacts collected a generation ago were dispersed or ended up in museums on the collector's death.

In the case of the Sahara, the region's inhabitants, driven by poverty, the lack of other economic opportunities and the need for money, know that buyers can be found for the ancient artefacts that can be found in the desert. So they go out and scour sites to obtain it. Some of this material may be sold directly to tourists visiting this region. Jeremy Keenan notes (2005; 2013: 225) "there is scarcely a settlement in the Sahara in which a local market selling such artefacts will not materialise within minutes of the arrival of tourists". The development of this market however is not only a result of the demands of collectors, for he says "I have witnessed tourists buying such objects in some of the most remote and impoverished corners of the Sahara simply because they feel that they should contribute to the alleviation of hardship, rather than for any desire to possess the items being sold". Keenan (2013: 225) adds that this activity is further stimulated by middlemen who have developed networks to the most distant desert communities to provide traders, agents and collectors in the main tourist centres with a supply of objets d'art for the souks, shops and hotel foyers of the tourist centres. He also reports that some local and European tour operators even offer artefact hunting excursions to known productive sites to visitors as part of the "desert experience" (and Di Lernia 2005: 448 notes cases of these sites being contaminated by their "seeding" with fake artefacts for tourists to "find").

The Sahara region, Morocco in particular, has since the 1980 os been a source of many high grade fossils and mineral specimens. Since $c$. 1997 it has also been a major conduit for the transit of meteorite finds surface reportedly gathered in the Sahara by nomads who learnt that western markets were interested in them and brought them to local middlemen and dealers by the sack and camel-load (Gilmer [2009]; Keats 20I4). It seems that they also learnt to gather arrowheads and other lithic items at the same time. This is probably the source of the stories frequently told by dealers who suggest that the prehistoric lithic material is "gathered by indigenous nomadic tribes during their travels across the Sahara Desert in Africa" to sell to earn money for supporting their families. The activity is thus depicted as economically beneficial for the populace of the developing countries.

Despite the image of supporting the desperately poor opportunistic nomads of the region that western dealers may prefer to project, it is clear that the trade is also being supplied by highly mobile professional European artefact hunters who enter the Sahara and scour archaeological sites for artefacts for sale (Keenan 2013: 22I). The features of the assemblages offered by several dealers seen in the 2019 survey suggested that this was the manner in which the items in their stock had reached the market. 
These activities have caused significant damage to the archaeological record of several regions of the Sahara. Keenan suggests (2013: 230) that although the precise number of artefacts looted from the Sahara is not known, it is an enormous amount, "for example, it is generally estimated that some two million artefacts have been looted from the Tassili region of Algeria alone". Savino Di Lernia (2005: 447) discussed the effects of this looting in Libya, noting the "astonishing impoverishment of the archaeological record" across the region, where over the last few years, sites have suffered from:

"continuous pillage of prehistoric artefacts: lithics, pottery and grinding stones have been almost entirely 'vacuumed up' from hundreds of sites, which were virtually untouched only a few years ago. Indeed, the popularity of these elements of the Sahara's cultural heritage has generated a growing appetite for their acquisition, with the result that there is a recognisable market based on the looting and theft of these prehistoric artefacts from their places of origin. African arrowheads, grinding stones, Acheulean hand-axes - to name but a few, are to be found in the stalls of European and North American markets [...and] on Internet sale sites".

Several dealers, observing the market, seem fully aware of the degree of this depletion. One writes in his sales offers on eBay: "in my opinion, this is one of the last frontiers in relic collecting, so add some of this material to your collection while it lasts". Another adds: "These pieces are getting harder and harder to come by [...] each batch I get I think it will be my last". ${ }^{1}$ Keenan reports (2003) that in the areas of the desert with which he is familiar, "vast regions of immense archaeological importance have been simply sterilised by systematic 'vacuuming' of artefacts". He gives (2013: 223) a thought-provoking example of a region that has been affected by the obliteration of "most of the best-known and accessible sites":

"For example, a reconnaissance of the most accessible terraces between the much publicised Temet dunes and Aïr (Niger) in November 2002, which had been liberally covered with Palaeolithic and Neolithic tools and pottery as recently as 1998, revealed that they had been thoroughly scoured: not a single stone tool or potsherd could be found. The same reconnaissance revealed that most of the western margin of the Tenéré desert appeared to have been equally well scoured".

Here it is worth noting that the Aïr and Ténéré Biosphere Reserve in the Agadez region of the north of Niger has been a World Heritage site since 1997.

\section{ANTIQUITISM AND LITHICS}

It is clear that through the antiquities trade, large numbers of freshly obtained "portable antiquities" (archaeological artefacts) are disappearing into innumerable scattered

1 eBay sellers $\mathrm{r} . . . \mathrm{O}$ and g...r. The last comment comes from the sale of a batch of Iooo assorted projectile points sold in February 2020 for 800 dollars. It is one of 20 batches of this size that the latter seller listed at the end of 2019. He sold at least two of them in a matter of weeks. 
ephemeral personal collections. Despite them providing the motor for the activity, the collectors themselves are largely invisible. Little is known about what they accumulate and how they actually go about using the material they buy. For many, these objects may function as something that makes the past tangible. They may carry an emotional charge, inspiring reflection on transience and yet the common humanity of generations long, long past. Probably many people buying these artefacts see them simply as something "cool", and unusual, and many most likely are sold to individuals that do not go on to become collectors.

Some collectors claim a more serious, academic interest in the items they collect. The dedicated collectors of portable antiquities often claim that by accumulating these items, they are not only "preserving them", but they are also engaging in the study of the past/past cultures. They represent both of these activities as somehow enlightened and beneficial (Saweged 1999; Atwood 2004: 31-33; Tokely 2006). The frequent argument that, by giving them a good home (McIntosh et al., 1995) by purchasing them, a collector is preserving the artefacts from some threat, ignores the fact that the loose objects derive from the destruction of an archaeological context, financed by the collectors' market itself.

The collectors of portable artefacts quite often see what they do with their finds as some kind of academic pursuit and represent themselves as independent (amateur or vocational) scholars. The study of loose decontextualised antiquities without even a known findspot as merely a heap of finds on a table has obvious limitations, and in the absence of (or disregard of the value of) contextual information, their handling ("antiquitism") is centred on subjective interpretation of the formal characteristics of the object.

In the case of Saharan lithics, there are relatively few publications written by such collectors, but one notable example is the book Flint Artefacts of North Africa (2005) by US collector Dave Greenwell. He realises that the production of regional typologies, is impossible "due to the way these artefacts have been collected [...] almost all provenance information has been lost forever. I don't offer this as a criticism, just as an observation". He does mention that collectors' lore associates some of the object types to particular regions. Some of them relate to pre-dynastic artefacts of the Nile Valley (see below), while he identifies some types that are said to be from Mali, Mauretania and Algeria. The book centres on producing "a methodological typology for Saharan Neolithic flint artefacts" and is organized in four sections. In the first ("a taxonomist's view") the types are organized in hierarchical dendrograms (with the typology derived from that used in the collecting of Native American lithics). The second gives a brief description of the characteristics and rarity on the market of each of the forms he identifies. Although it is called "an academic's view" there is nothing academic about it. In the book's third and fourth parts he illustrates selected points with colour photos ("the artist's view") and attempts a superficial account of the raw materials ("a geologist's view"). The bulk of the items classified are 55 types of Neolithic arrowheads, but 
316 Discussions and criticism

also some small tools (drills/borers, items termed "gravers", scrapers, crescent knives and discs). One group of unifacial points is differentiated (Greenwell 2005: 44-46) and labelled "Mesolithic". There are no axes/celts/gouges or other pecked and ground tool types mentioned. The book makes no reference to any outside literature, with no references to any of the archaeological literature existing at the time.

Very similar in content is the expensive self-published and self-justificatory coffeetable book Sahara: Material Culture of Early Communities, Prehistoric Artifacts (Klenkler et al., 2016). This is another collectors' attempt to imitate scholarship through merely typological sorting and narrativisation of decontextualised material. The authors cannot give the findspots of the finds they illustrate (but claim that this is to protect the sites from which they came from looting). This book received a detailed review by two French scholars (Vernet and Le Quellec 20I7) who are scathing about its approach, methods and failure to use modern literature in attempting to present a collectors' picture of prehistory:

Ce type de classification ne présente donc strictement aucun intérêt... sauf pour les acheteurs de pointes de flèches qui se demandent comment ordonner leurs acquisitions dans leurs vitrines. [...] cet ouvrage s'apparente à un catalogue de salle de vente sur lequel on aurait omis d'indiquer les prix. La passion typologique dont il témoigne est typique des collectionneurs ignorant tout du contexte culturel des objets qu'ils ont amassés, mais qui veulent néanmoins les classer. ${ }^{2}$

They end by noting that this book is similar to a genre that has recently become common: pseudo-manuals intended to be used as reference books by "collectors who spend their leisure time searching for artefacts on archaeological sites or buying them on the Internet", and noting with regret that the modern demand for works of this kind is such that they can become bestsellers, constantly reprinted, with a circulation that can sometimes exceed 50,000 copies.

\section{A LOOK AT THE TRADE IN SAHARAN NEOLITHIC MATERIAL}

There are many online venues where Palaeolithic to Neolithic artefacts originating in North Africa may be obtained, ranging from specialist antiquities dealers with their own internet shops to major public media outlets such as eBay or Facebook or several dedicated portals such as Sixbid, Catawiki, V-coins and many local sites (such as Allegro

\footnotetext{
2 "This type of classification is therefore of absolutely no interest... except for buyers of arrowheads who want to know how to order their acquisitions in their showcases [...] This work is similar to a salesroom catalogue in which the prices have been omitted. The typological passion it exhibits is typical of collectors who are unaware of the cultural context of the objects they have collected, but who nevertheless want to classify them". Translated by Author.
} 
in Poland). A detailed search carried out in August 2019 indicated that a large amount of Saharan lithic material was being openly offered on the internet.

This presentation will concentrate on only part of this global trade, namely the sales conducted via the longest established and largest, eBay. This is because the information available about the sales and dealers on other sites could not be presented in a consistent format to allow analysis. EBay has over 90 million users and annually sells some 700 million objects of all types. Table I presents the results of a search for Saharan Neolithic material carried out, from 5th to 9th August 2019, on eBay.com and its semi-independent national services in the UK, Canada, Australia, France, Spain, Germany, Holland, Switzerland, Belgium, Italy, Poland and Russia. This material was located by using a variety of search terms in various languages in the portal's own search engine, and the results were manually processed to avoid duplication. Results were also sought from the eleven countries that cover parts of or are adjacent to the Sahara desert (Algeria, Chad, Egypt, Libya, Mali, Mauritania, Morocco, Niger, Sudan, Tunisia and Western Sahara). The other objects being offered by each seller identified were also examined, to determine the place of Saharan Neolithic material in their offers. Their feedback was analysed, in order to examine the history of recent transactions. The research was quite time-consuming, eBay is deliberately constructed to maintain buyer and seller anonymity and obscure a number of features about transactions, both those in progress as well as those that took place in the recent past. In the first week of 2020, the same sites and sellers were revisited, to see whether the situation had changed six months later. In fact few changes were observed, which suggests that the 2019 survey can be taken as representing a snapshot of the current state of this part of the market.

\section{Dealers}

The distribution of the sellers is shown in Figure I (which shows non eBay sellers too). Most of the Saharan Neolithic material is being redistributed by dealers operating through eBay.com (the main US-based one, 22 sellers) and eBay.uk (9 sellers). On eBay Canada and Australia, there were three and two sellers respectively. Among the European eBay portals, the French one with ten sellers was the most involved in this trade. The local eBay portals for Belgium and Holland had none and the Spanish and Italian ones two each. The German one had seven sellers. It is notable that the Austrian one had just one seller and the Swiss one none. This is significant because from Ist July 2008, the German, Austrian and Swiss portals of eBay introduced new regulations concerning the sale of archaeological artefacts. They must be accompanied by proper documentation showing the seller's title, and that the correct procedures for reporting and export have been followed. Interestingly, one of the biggest German dealers avoids this by listing his Saharan Neolithic artefacts in the section for rocks, minerals and fossils (in the "Mammut" subsection). In August 2019, there was a total lack of Saharan Neolithic material from the eBay portals in eastern Europe, including the Russian one (which does contain auctions of local Stone Age material). 
In Table I, the screen names of dealers have been anonymised (in the same way that eBay uses to protect buyer identity). They are organized by the size of their feedback, suggesting their overall volume of trade and longevity. Their location is listed in the second column (using the standard 2-digit USP abbreviations for states in the USA and the 3-digit ISO country codes elsewhere). Column three gives first the number of Saharan lithic items being sold at the time of the survey, and then the total number of objects on sale by that dealer at the same time; this gives an idea of the relative importance of the material under discussion to the dealer's whole offer. The fourth column contains abbreviations (arranged in order of prominence in the sales offers) denoting other types of material being handled by this dealer. ${ }^{3}$

The sixty or so sellers seem to belong to a number of groups (cf. Fay 2013: 197). One consisted of individuals offering a small number of Saharan lithic items in an offer that consists mostly of a wider variety of general collectables or unwanted household items. Their feedback on past transactions may or may not exhibit traces that they have sold other such items in the past. Rather than as a result of a concerted collecting action, it is probable that the Saharan Neolithic artefacts offered have come from the incidental purchases (for example at a rocks and minerals fair) of a few items as "something cool to have" that were now being disposed of, or maybe these were items picked up at an estate sale, or perhaps bought on speculation for resale.

Most of the sellers seem to be dealers trading in various collectables, such as mineral specimens, fossils, meteorites, ethnographic items and along with them they have moderate quantities of Saharan lithic items in their stock. Some seem to have got their specimens from the same area (in the case of rocks and minerals, most often it seems Morocco), others have stocks that have been obtained from different worldwide sources. There is a smaller group of dealers that (while occasionally offering other items) specialise in the sale of Saharan lithics and have relatively large assemblages of this material in stock with high volume sales. These dealers also sell other items alongside the stone tools (minerals, North African ethnographic collectables etc.). It is possible that some of these dealers are in fact collectors, who buy in bulk in order to obtain the best specimens and then sell the surplus to cover their costs.

It is notable (Fig. I) that, the sellers are for the most part based in the US, the UK and several western European countries. This is not surprising, the US and UK are both major players in the global art and antiquities market (Fay 2013: 197). The distribution in the US corresponds to that of dealers and collectors of ancient artefacts

3 These are as follows: $\mathrm{M}$ - rocks, fossils and minerals; N - Native American artefacts; E - other ethnographic objects and art; $\mathrm{Z}$ - general collectables (sports cards, antique items, modern coins, paper money, postcards etc.); A - portable antiquities; $\mathrm{C}$ - ancient and medieval coins; $\mathrm{B}$ - collectable (antique) beads other than Saharan Neolithic ones; J - modern and collectable jewellery; D - household items and bric-a-brac; $\mathrm{O}$ - other. 


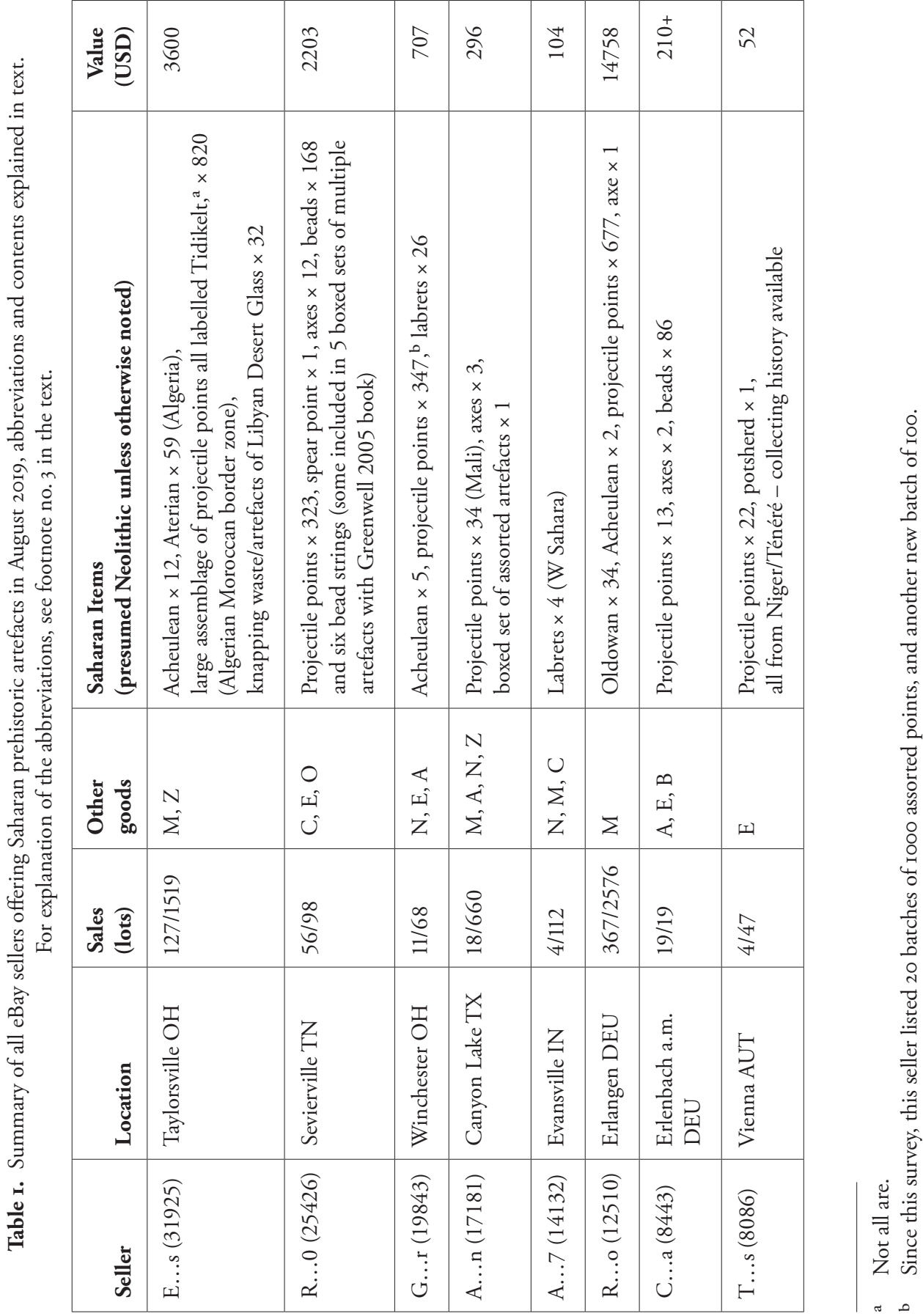


320 Discussions and criticism

\begin{tabular}{|c|c|c|c|c|c|c|c|c|c|c|c|c|c|c|}
\hline 䓻 & $\begin{array}{l}\stackrel{+}{0} \\
\text { 岌 }\end{array}$ & 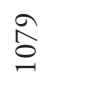 & $\stackrel{8}{ }$ & $\underset{\forall}{\stackrel{ }{*}}$ & $\begin{array}{l}\stackrel{\infty}{\stackrel{1}{\rightleftharpoons}} \\
ٍ\end{array}$ & $\stackrel{n}{n}$ & $\stackrel{0}{=}$ & $\underset{f}{\stackrel{f}{n}}$ & తి & $\approx$ & 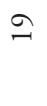 & $\hat{\widehat{n}}$ & $\underset{\infty}{\infty}$ & m \\
\hline 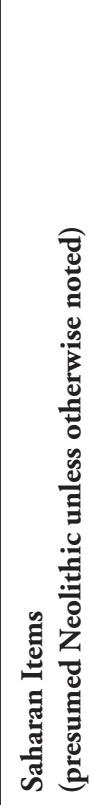 & 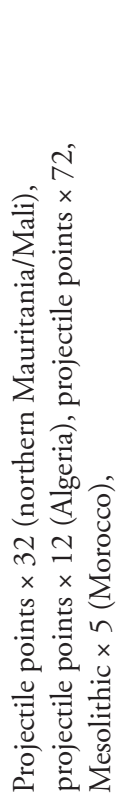 & 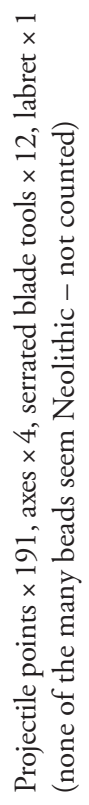 & 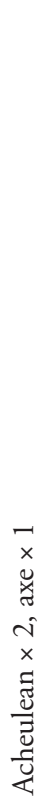 & 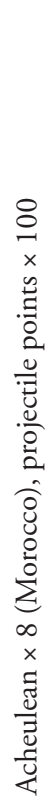 & 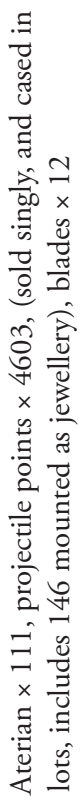 & 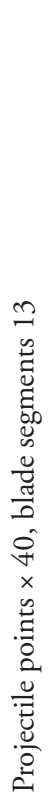 & 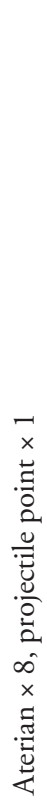 & 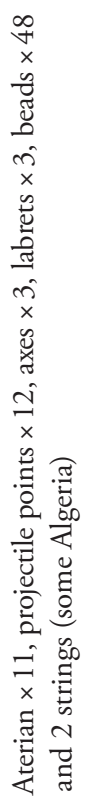 & 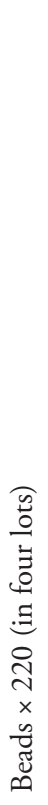 & 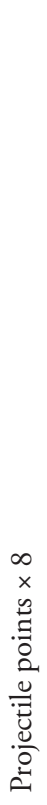 & 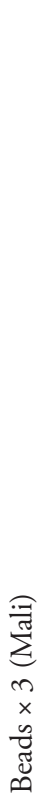 & 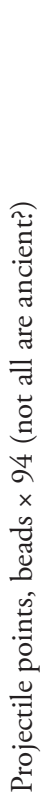 & 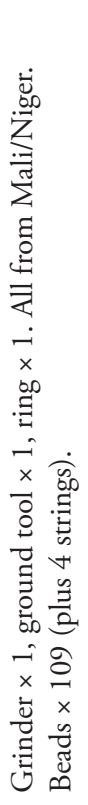 & $\begin{array}{l}\sim \\
x \\
\ddot{\ddot{u}} \\
\ddot{x}\end{array}$ \\
\hline 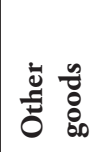 & $\begin{array}{l}n \\
\text { मी } \\
\hat{4}\end{array}$ & $\begin{array}{l}\hat{\Sigma} \\
\hat{n} \\
\hat{n} \\
\hat{-1}\end{array}$ & $\Sigma$ & $\Sigma$ & $\begin{array}{l}0 \\
\hat{u} \\
\hat{Z} \\
\hat{\sim}\end{array}$ & $u$ & $\begin{array}{l}n \\
\hat{N} \\
\hat{n}\end{array}$ & $\stackrel{n}{z}$ & صิ & $\begin{array}{l}0 \\
\hat{u} \\
\hat{N}\end{array}$ & 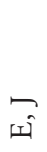 & 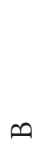 & 디 & \&ी \\
\hline 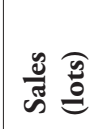 & $\frac{\varrho}{a}$ & $\begin{array}{l}\sqrt{\sigma} \\
\stackrel{\hbar}{\nexists} \\
=\end{array}$ & $\frac{\infty}{m}$ & $\frac{\sqrt{n}}{a}$ & 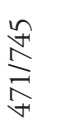 & $\frac{i}{n}$ & 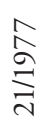 & $\frac{n}{n}$ & $\stackrel{m}{\curvearrowright}$ & $\begin{array}{l}\frac{\infty}{\varpi} \\
\frac{\sigma}{m}\end{array}$ & 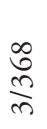 & 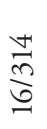 & 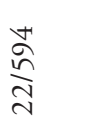 & 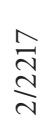 \\
\hline & 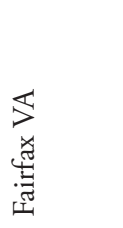 & 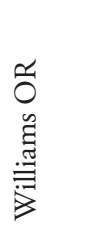 & 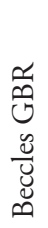 & 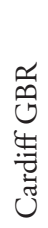 & 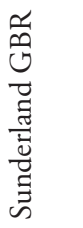 & 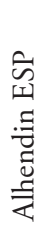 & 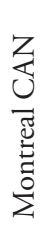 & 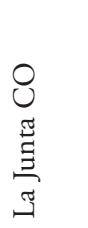 & 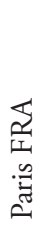 & 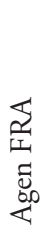 & 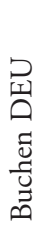 & 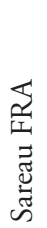 & $\begin{array}{l}\mathbb{U} \\
0 \\
0 \\
0 \\
0\end{array}$ & 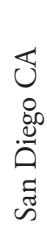 \\
\hline$\stackrel{\bar{\nu}}{\bar{\nu}}$ & 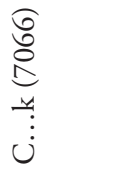 & 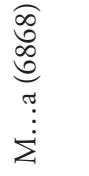 & 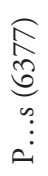 & 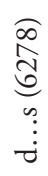 & 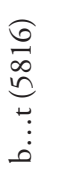 & 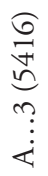 & 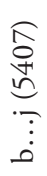 & 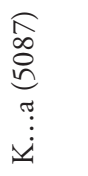 & 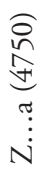 & 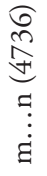 & 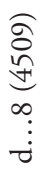 & 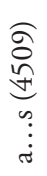 & 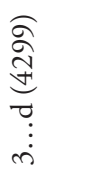 & 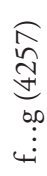 \\
\hline
\end{tabular}




\begin{tabular}{|c|c|c|c|c|c|c|c|c|c|c|c|c|}
\hline 总会 & $\stackrel{\smile}{\sim}$ & $\stackrel{\star ָ}{\sim}$ & $\begin{array}{l}\stackrel{̊}{\Xi} \\
=\end{array}$ & $\stackrel{\leftarrow}{n}$ & $\stackrel{\varpi}{m}$ & 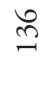 & ñ & 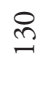 & $\vec{n}$ & ํํำ & ฉ & $\stackrel{尺}{I}$ \\
\hline 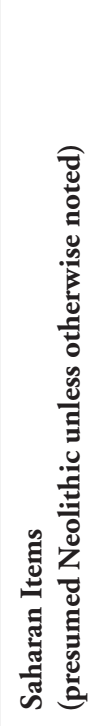 & 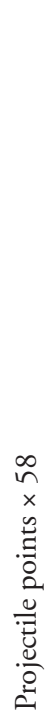 & $\begin{array}{l}n \\
x \\
y \\
\ddot{y} \\
\dot{y}\end{array}$ & 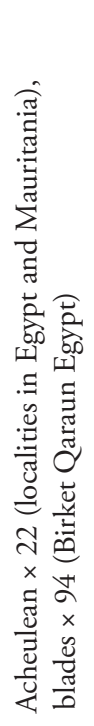 & 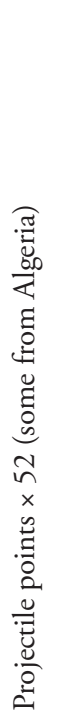 & 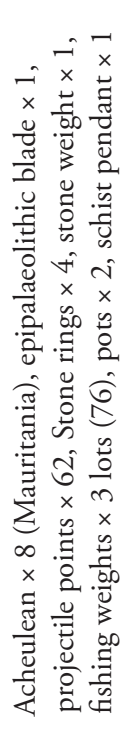 & 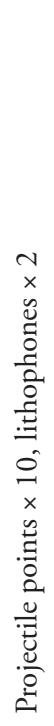 & $\begin{array}{l}\infty \\
x \\
\tilde{z} \\
\tilde{J} \\
\infty\end{array}$ & 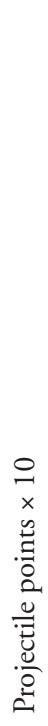 & 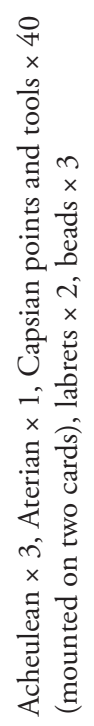 & 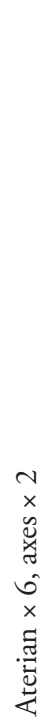 & 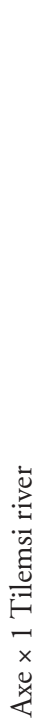 & 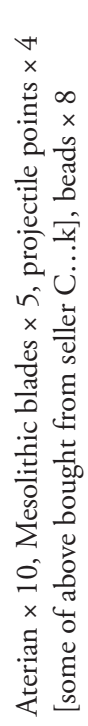 \\
\hline 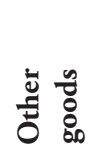 & $\begin{array}{l}N \\
\bar{Z}\end{array}$ & $\begin{array}{l}u \\
\dot{\Sigma}\end{array}$ & $\begin{array}{l}n \\
\dot{z} \\
\dot{z} \\
\hat{u}\end{array}$ & $\begin{array}{l}\hat{\Sigma} \\
\dot{\sum} 0 \hat{N}\end{array}$ & $\stackrel{\varphi}{\ddot{<}}$ & $\Sigma$ & $\varangle$ & $\begin{array}{l}0 \\
\dot{\Sigma} \\
\dot{\Delta} \\
\hat{i}\end{array}$ & $\varangle$ & $\Sigma$ & $\varangle$ & $\begin{array}{l}z \\
\dot{z} \\
\hat{u}\end{array}$ \\
\hline$\frac{\mathscr{b}}{\tilde{m}} \stackrel{\widehat{p}}{0}$ & $\stackrel{n}{=}$ & $\frac{n}{\frac{7}{m}}$ & 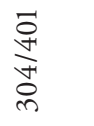 & $\frac{\mathfrak{\infty}}{\stackrel{\infty}{\sigma}}$ & $\underset{\substack{0 \\
\stackrel{D}{N}}}{\stackrel{b}{N}}$ & $\hat{\lambda}$ & 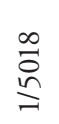 & $\frac{\infty}{\sigma}$ & 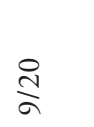 & $\frac{N}{\infty}$ & 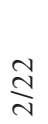 & $\frac{\infty}{n}$ \\
\hline 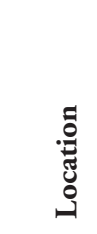 & $\begin{array}{l}\vec{\Sigma} \\
0 \\
0 \\
0 \\
0 \\
0\end{array}$ & 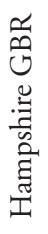 & 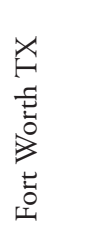 & 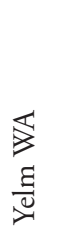 & 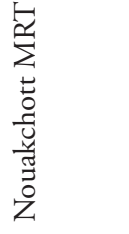 & 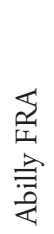 & 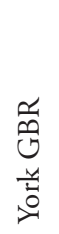 & 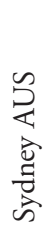 & 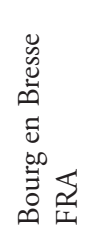 & 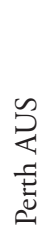 & 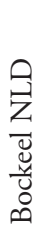 & 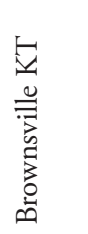 \\
\hline 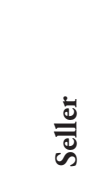 & 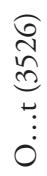 & 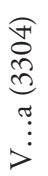 & 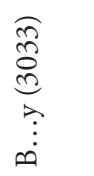 & 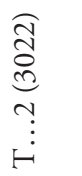 & 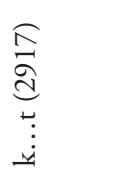 & $\begin{array}{c}\widehat{\widetilde{n}} \\
\tilde{\widetilde{d}} \\
\vdots \\
\vdots \\
\vdots \\
0\end{array}$ & 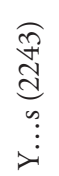 & $\begin{array}{c}\underset{\hat{\partial}}{\infty} \\
\stackrel{\infty}{\rightleftarrows} \\
\vdots \\
\vdots \\
\&\end{array}$ & $\begin{array}{c}\widehat{\approx} \\
\hat{n} \\
\\
\vdots \\
\vdots \\
\Sigma\end{array}$ & 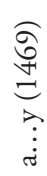 & 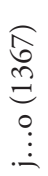 & 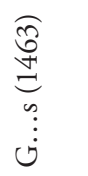 \\
\hline
\end{tabular}


322 Discussions and criticism

\begin{tabular}{|c|c|c|c|c|c|c|c|c|c|c|c|c|c|c|}
\hline 异 & $\hat{n}$ & 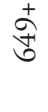 & $\Lambda$ & ๖े & $\stackrel{\infty}{\sim}$ & $\stackrel{0}{\sigma}$ & $\ddot{\sim}$ & $\stackrel{\infty}{\stackrel{\varpi}{m}}$ & 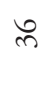 & $\frac{N}{m}$ & $\cong$ & $\frac{N}{6}$ & $\stackrel{\infty}{\wedge}$ & $\stackrel{\ominus}{\curvearrowleft}$ \\
\hline 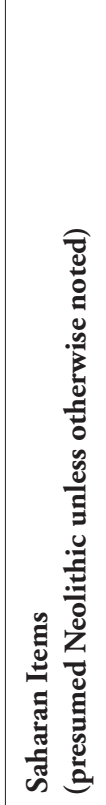 & 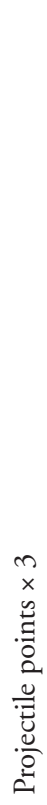 & 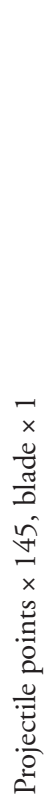 & 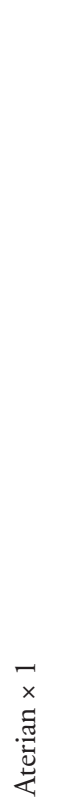 & 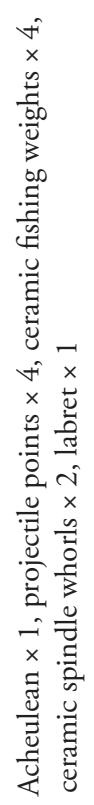 & 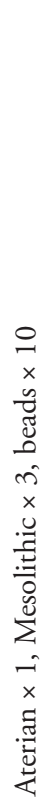 & 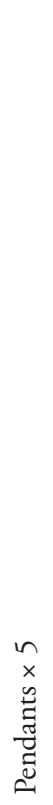 & 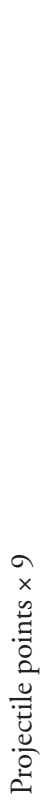 & 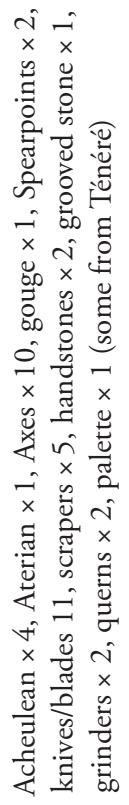 & 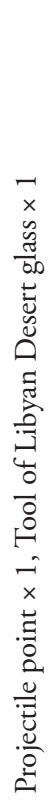 & 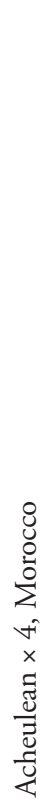 & $\begin{array}{l}\vec{x} \\
\ddot{y} \\
\vec{x}\end{array}$ & 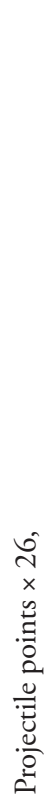 & 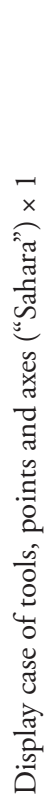 & 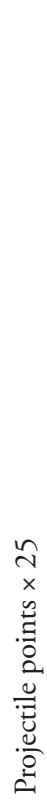 \\
\hline 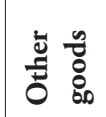 & $\varangle$ & $\Sigma$ & $\begin{array}{l}\sum_{j} \\
N\end{array}$ & $\begin{array}{l}0 \\
\hat{u} \\
\hat{\&}\end{array}$ & $\begin{array}{l}\sum \\
N\end{array}$ & $\begin{array}{l}\mathbb{Z} \\
\infty \\
=\end{array}$ & $\begin{array}{l}\text { II } \\
\dot{z} \\
\hat{\theta}\end{array}$ & $\Sigma$ & 디 & « & $\begin{array}{l}\text { II } \\
\hat{N} \\
\hat{E}\end{array}$ & $\begin{array}{l}Z \\
\dot{Z}\end{array}$ & $\stackrel{\Xi}{N}$ & $\varangle$ \\
\hline$\frac{0}{\tilde{n}} \frac{\hat{a}}{0}$ & $\frac{n}{n}$ & $\frac{\nwarrow}{\infty}$ & $\triangleq$ & 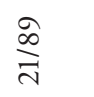 & 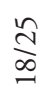 & $\frac{2}{n}$ & $\stackrel{n}{\lambda}$ & $\underset{\forall}{\stackrel{\nabla}{F}}$ & $\stackrel{\stackrel{d}{d}}{\sim}$ & $\underset{\ngtr}{\stackrel{\overbrace{}}{\ngtr}}$ & $\underset{+}{\stackrel{t}{=}}$ & $\frac{m}{n}$ & $\stackrel{\varpi}{\sim}$ & $\frac{n}{n}$ \\
\hline & 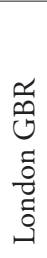 & $\begin{array}{l}\text { aै } \\
0 \\
0 \\
0 \\
0 \\
0 \\
0 \\
0 \\
0\end{array}$ & 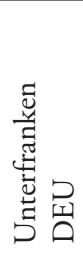 & 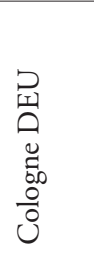 & 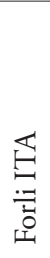 & 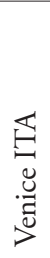 & 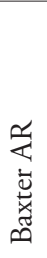 & 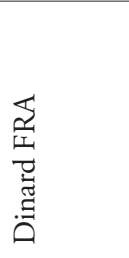 & 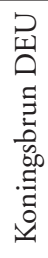 & 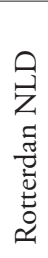 & 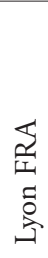 & 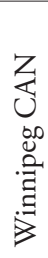 & 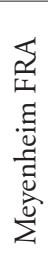 & 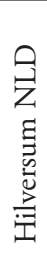 \\
\hline$\frac{\ddot{\Xi}}{\bar{E}}$ & 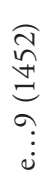 & 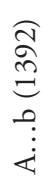 & $\begin{array}{c}\underset{\mathbb{J}}{\Xi} \\
\vdots \\
\vdots \\
0\end{array}$ & 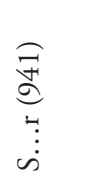 & 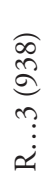 & 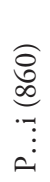 & $\begin{array}{c}\text { Oิ } \\
\text { } \\
\infty \\
0 \\
\vdots \\
\vdots\end{array}$ & $\begin{array}{c}\widehat{\partial} \\
\stackrel{n}{\Xi} \\
-! \\
\vdots \\
\dot{n}\end{array}$ & 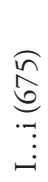 & $\begin{array}{c}\sqrt{\tilde{n}} \\
\underset{6}{6} \\
\vdots \\
\vdots \\
0\end{array}$ & 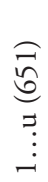 & 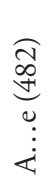 & $\begin{array}{c}\widehat{\Phi} \\
\underset{+}{ \pm} \\
0 \\
\vdots \\
\dot{0}\end{array}$ & 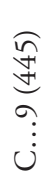 \\
\hline
\end{tabular}




\begin{tabular}{|c|c|c|c|c|c|c|c|c|c|c|c|c|c|c|}
\hline 芑会 & సิ & $\stackrel{\star}{\sim}$ & $\underset{\sim}{\approx}$ & तี & $\stackrel{\text { 工 }}{ }$ & ్․ & $\stackrel{\Re}{\wedge}$ & $\stackrel{\curvearrowleft}{\varrho}$ & ஜ્ & $\tilde{n}$ & 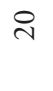 & $\stackrel{0}{ }$ & 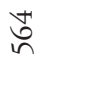 & \\
\hline 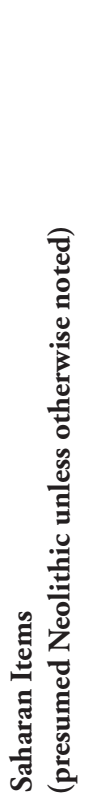 & 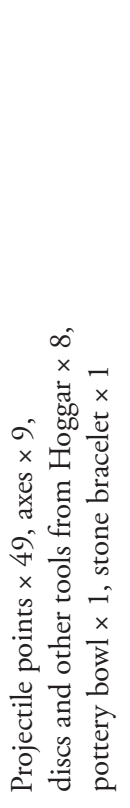 & 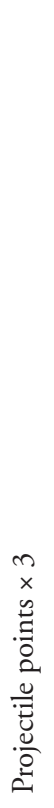 & 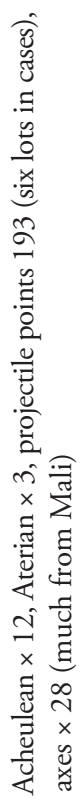 & 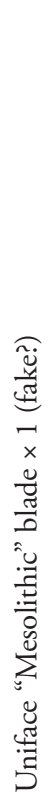 & 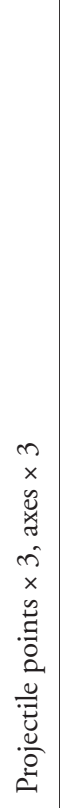 & 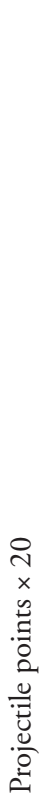 & 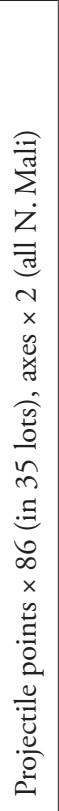 & 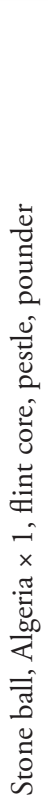 & 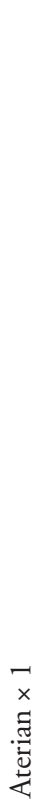 & 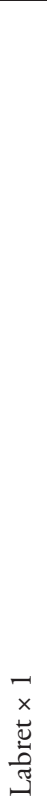 & 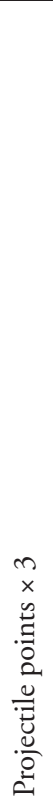 & 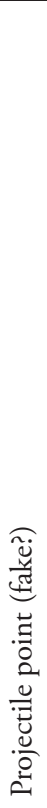 & 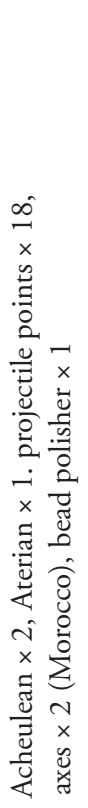 & 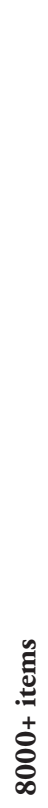 \\
\hline مُ & « & $\begin{array}{l}0 \\
\hat{N}\end{array}$ & $\begin{array}{l}\mathbb{N} \\
\hat{N}\end{array}$ & $\begin{array}{l}Z \\
\hat{u}\end{array}$ & $\begin{array}{l}\text { 디 } \\
\hat{N}\end{array}$ & $N$ & & $\begin{array}{l}\tilde{Z} \\
\dot{\Sigma}\end{array}$ & $\begin{array}{l}\varangle \\
\hat{N} \\
\hat{N}\end{array}$ & $\hat{n}$ & $\begin{array}{l}u \\
\hat{N}\end{array}$ & $\Sigma$ & $\cup$ & \\
\hline$\frac{0}{\frac{y}{0}}$ & $\underset{\infty}{\stackrel{N}{\infty}}$ & 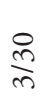 & 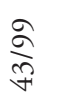 & $\stackrel{\infty}{\stackrel{\infty}{=}}$ & 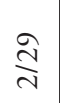 & $\stackrel{\gtrsim}{ঔ}$ & $\frac{n}{n}$ & 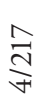 & $\stackrel{\infty}{\stackrel{N}{=}}$ & $\stackrel{n}{‡}$ & $\frac{n}{n}$ & $\stackrel{\cong}{\triangleq}$ & $\underset{\substack{\infty \\
\sim}}{\stackrel{\infty}{N}}$ & \\
\hline 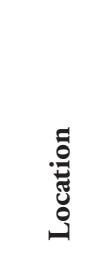 & 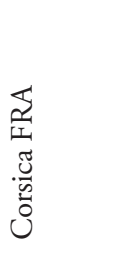 & 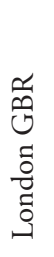 & 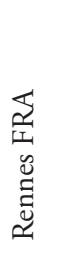 & 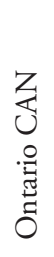 & 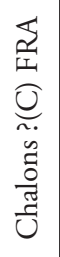 & 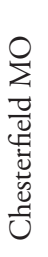 & 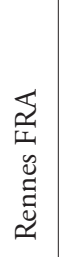 & 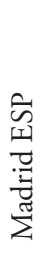 & 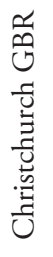 & 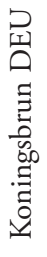 & 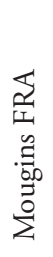 & 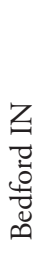 & 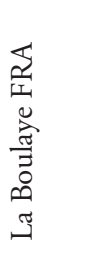 & \\
\hline 离 & $\begin{array}{c}\widehat{n} \\
\hat{n} \\
\vdots \\
\vdots \\
\vdots \\
\end{array}$ & 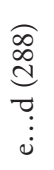 & 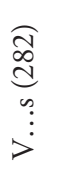 & $\begin{array}{c}\underset{\hat{n}}{d} \\
\underset{d}{0} \\
\vdots \\
\vdots\end{array}$ & 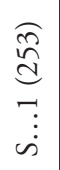 & 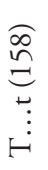 & 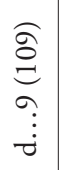 & 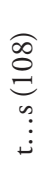 & 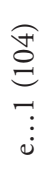 & $\begin{array}{c}\widehat{\approx} \\
\underset{n}{n} \\
\vdots \\
\Xi\end{array}$ & 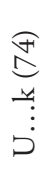 & 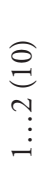 & $\begin{array}{c}\widehat{\Xi} \\
-\vdots \\
\vdots \\
0\end{array}$ & قّ \\
\hline
\end{tabular}




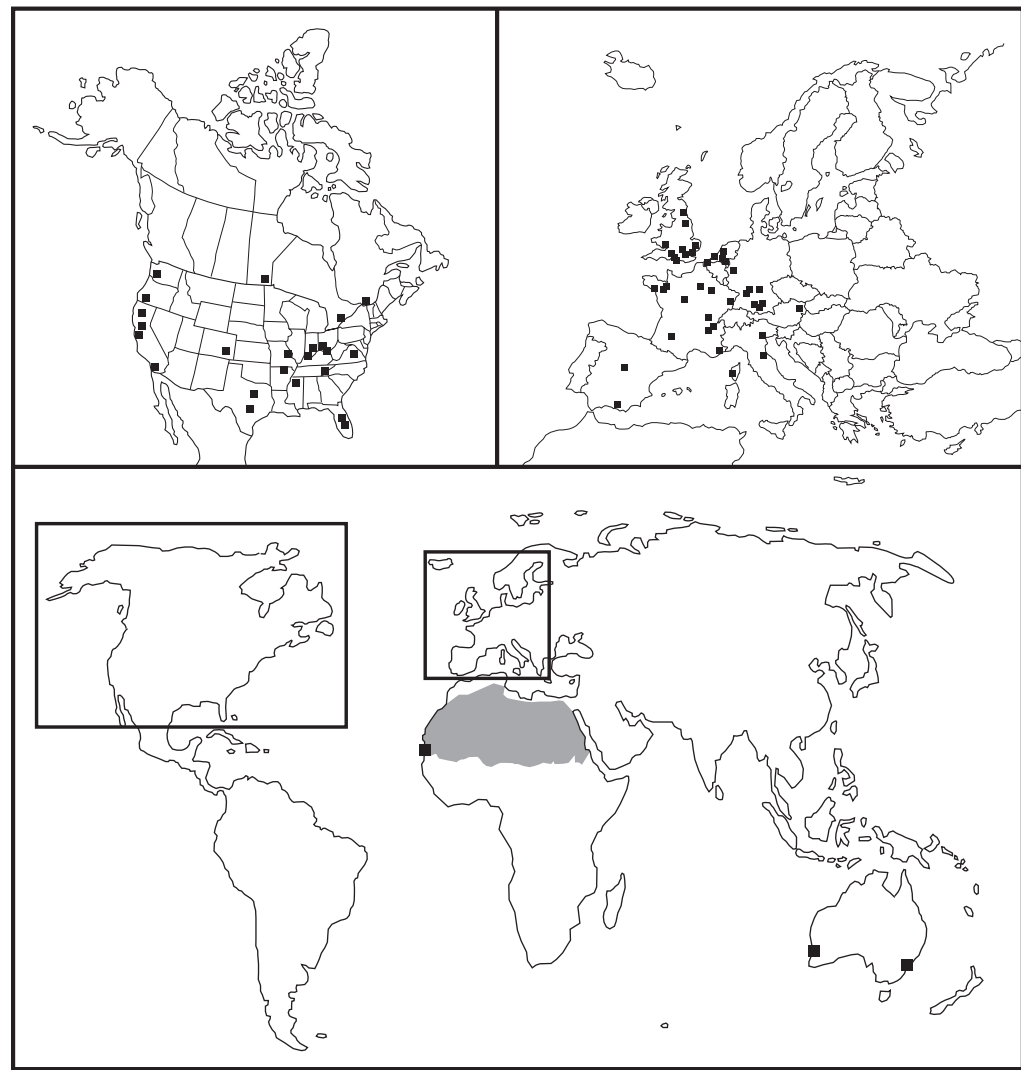

Fig. I. Map showing global distribution of all online sellers (not only on eBay) offering Saharan prehistoric artefacts in August 2019. Drawn: M. Kosińska.

(including coins) in general. This to some extent matches the distribution of population and wealth. The same applies to the concentrations of dealers in the richer areas of Germany, Britain, France and the Low Countries. It should be noted that only one of these dealers is based in the source countries. It would be problematic to sell ancient artefacts abroad if a country does not allow such export, and it is interesting to note that this seller has some arrangement whereby the artefacts are posted from France, thus avoiding direct export straight to the customer.

\section{Buyers}

We know very little about the buyers, whose identity and location are known only to the seller. Their own comments in feedback mainly refer to the ease and reliability of the transaction, or the bargain price. One or two reveal something of their attitude 
concerning what they have bought: "Very pleased with this - it is a display quality object. Thanks", "beautiful objects, outstanding", "awesome!", "awesome artifacts", "A+A+A+little buggers they are", "fantastic value for something this age", "this item is just what I wanted for a good price", "Jim patiently answered all my questions, and I got exactly what I wanted!", "A knowledgeable collector and a pleasure to work with", "Really a great authentic artifact - thanks a lot!", "excellent relics", "well packaged and exactly as described. My students will love touching them", "perfect, I felt like I timetravelled holding it $\odot$ Thanks, will buy again”. Occasionally, it seems that one seller had bought an item from another and then tried to resell it at a higher price.

\section{Authenticity}

The expanding global antiquities market has to cope with a situation where a market exists for material that is becoming more difficult to obtain as the most accessible and productive sites become depleted. The demand is often met by producing fake artefacts. Many archaeologists are aware that the internet market in antiquities is full of fakes (Stanish 2009; Fay 20II; Brodie 20I5: II), and it is a major concern of buyers to avoid getting caught out. The market for prehistoric stone tools is no exception to this general rule. It is difficult to use the photos and descriptions in sales offers unequivocally to determine whether a given artefact is a fake. Bad fakes stand out easily, while the best ones will deceive even the most discerning collectors. There are a number of articles in the amateur literature connected with arrowhead collecting in the US that give pointers to identifying fakes (but they will not be rehearsed here because repetition would make it easier for the fakers to perfect their craft).

There is probably somewhere in North Africa a workshop that is turning out considerable numbers of "Acheulean handaxes" in a biscuit-coloured laminated sandstone, and then apparently given a "desert patina" by sandblasting (possibly in a car bodywork workshop). They are mainly sold by one dealer but several other dealers have similar looking objects that might have come from the same supplier. In the case of the Aterian points, one may observe that quite a lot seem to have been retouched after being affected by deep patination. Although this effect has apparently been observed in "grounded" (archaeologically obtained) artefacts too, in the case of material that has freshly surfaced on the market, it seems more likely that this was an attempt to "improve" a poorlyshaped item to make it more saleable. Some dealers specifically note that their material has not been retouched, showing that this is something the buyer ought to be wary of.

When it comes to the artefacts relating to the Neolithic Green Sahara period, it seems that the relative quantities of dubiously-authentic material are not as great as in the case of US arrowhead collecting (Hothem 1992; Whittaker and Stafford 1999; Berner 2002). It does seem, however, that there are a number of skilled flint-knappers operating in the Sahara region producing some very passable ancient-looking artefacts. One high-volume seller has an interesting range of lithic items, many of which are 
326 Discussions and criticism

well-crafted and attractive (including for the colour and pattern of the stone), but although it seems he has some artefacts that do look authentic, there are quite a lot of others that have a number of characteristics that suggest, whatever they are represented as being, that they are modern products made for the market. There are a number of other sellers that also have similar fresh-looking material (see also Di Lernia 2005: 448).

It may be that not all of the dealers selling these fakes are knowingly engaging in fraudulent activity. In some cases, the fake items occur on sale alongside other material that seems authentic. Dealers that do not have specialist knowledge of the field may have bought these items on the understanding that this was all freshly looted from archaeological sites, when in fact real artefacts had been bulked out with fakes (this apparently happens a lot in other areas of the antiquities market). It is interesting to note that in one case examined in the 2019 survey, it seems it was apparently genuine Saharan arrowheads that were being added to improve a range of sales otherwise consisting entirely of fakes representing antiquities from a variety of old world cultures.

\section{Comments on the Categories of Artefacts Sold}

The fifth column in Table I gives a summary of the various types of object being sold as prehistoric artefacts by these sellers, they are described below. In the next column is the total value assigned to them by the sellers (the great majority of them were fixedprice Buy-it-Now items, where there was bidding, the price was the bid at the end of the week). Beads will be discussed separately below.

\section{Palaeolithic Lithics}

There were two main groups of Palaeolithic material sold. There were some primitive tools that were described as Oldowan, although the photos however suggest that not all of these items had clear evidence of human manufacture. The second group were a larger number of handaxes $(87)$ that were labelled Acheulean. Again, not all of them seem to be genuine ancient artefacts. In both cases, the general locality where they had been found was reported; they had come from a wide range of places across the region.

\section{Middle Stone Age}

The Middle Stone Age was represented mainly by 2I4 "pedunculate points" assigned to the Aterian technocomplex. Some "Aterian discs" were also noted, but no other artefact types. Most of these are sold individually, but some bulk lots also appear. They mostly seem to have been made of chert or flint and many are rather poorly-made. The objects tend to be heavily-patinated. The sellers seem unsure of the dating, giving a variety of $\mathrm{BP}$ and $\mathrm{BC}$ dates: $30 \mathrm{ka}+, 40 \mathrm{ka}, 120-55 \mathrm{ka}, \mathrm{I2O}-30 \mathrm{ka}, \mathrm{I} 45-20 \mathrm{ka}$ (the dates given in Wikipedia). The Late Palaeolithic is represented only by a few points (including some of "Ounanian" type). A few items (points and blades) labelled "Mesolithic" were being sold by several dealers. 


\section{Neolithic Lithics}

The bulk of the material revealed by this survey comprises Neolithic flints, associated by sellers with the "Green Sahara" (Neolithic Humid Phase) phase. Here too a wide variety of dates is cited by the sellers: IO.2-2k BC, 9-4 ka, 7-5 ka, 7-3 ka, 6-3 ka, 5-3 ka, c. $5000 \mathrm{BC}$, c. $4000 \mathrm{BC}$, "around $4000 \mathrm{BC}$ ".

The most prominent component ( $98 \%$ ) of this material consisted mainly of small projectile points, mostly complete (only a few sellers have cheap bulk lots of fragments, probably broken ones were removed before the left the source country). Sellers tend to market these singly (in which case they often put them in a small box with transparent lid and a generic label saying what it is), but most commonly they are sold in groups of between five and 50 items. Again, these may be mounted in display cases.

There is a wide variety of typology, though the examples seen in 2019 were mostly of the types designated "common" by Greenwell (2005; see also Hugot 199I). The groups of points offered by individual dealers tended to have different formal and technological characteristics, presumably due to the material coming from different regions and cultural contexts. Since any information about their origins and collection history has been obliterated by their passing through the market, no attempt was made to document this. Although many sellers give only a rough idea of size, the majority of the points are relatively small. Examples from $15-20 \mathrm{~mm}$ long are not uncommon, the majority seem to be $30-40 \mathrm{~mm}$ and examples $50-60 \mathrm{~mm}$ long are less common in the marketed assemblages. These points seem from the photos (for descriptions are rare) to have been mainly made of opaque chert or flint, or translucent flint of varied colours. Chalcedony, quartzite, and jasper were also occasionally used. Sellers frequently photograph the objects to show the craftsmanship and elaborate technology of the flaking, one of the attributes making them collectable. It can be seen that many of them are finely-made and the majority thinned and finished by extensive pressure-flaking. Some effort seems to have been put into creating points of high quality with specific desired characteristics. Some examples have serrated edges. They do not all seem particularly sharp, which raises the question of the function of such small points. As noted above, even though these items are generally sold very cheaply, there seem to be fakes on the market (cf. Di Lernia 2005: 448), but their extent is not clear. Apart from a few larger points that tend to be termed "spear heads", and a few discoidal knives, there were very few knapped flint tools being sold.

\section{Neolithic "axes"}

A number of sellers have typical Neolithic polished axes, referred to variously as "celts" or "axes", some merely as "tools" (but also some are termed "scrapers"). Some would classify as "gouges", but that is a term not seen very frequently in the online sales. Rarely is the material identified. The photos showed that many were of igneous rocks (basalt, dolorite, diorite, aplite and granite were mentioned), some seem to have been 
quartzite, while more common were those of silaceous materials (labelled chert and flint by the sellers). Many are ground all over, but some are only partially ground with extensive areas of flaking visible. The size ranges of the ones on offer clustered fairly equally into three groups $30-60 \mathrm{~mm}, 85-105 \mathrm{~mm}$ and $155-218 \mathrm{~mm}$ long, with only about IO\% of them between IIO and I $40 \mathrm{~mm}$. There a few "miniature" examples under $25 \mathrm{~mm}$ long and an outlier at $240 \mathrm{~mm}$ (total number of axes 92). It is difficult to determine from the photos if any of these items are modern fakes.

Other ground and pecked stone tools occurred only occasionally, and were priced accordingly, they include so-called "handstones" (of varying morphology and uncertain function), pestles, grinders and pounders, the occasional quern, perforated discs (weights?) and two items labelled lithophones. Possibly the infrequency with which they appear online is related to their weight and bulkiness (important if the artefact hunter is hand-carrying their haul while flying out of the region).

\section{Labrets}

Several sellers had smooth polished cylindroid artefacts and their fragments that they labelled labrets (also ear spools and lip plugs/pins) and dated to the Neolithic period. They were of varying sizes and shapes and many were made of polished quartz or chalcedony. Two separate items recently on sale online are pictured with what appears to be heavily corroded copper or iron wire wrapped around one end, which suggests that these examples at least are not Neolithic (or from the desert). While Neolithic labrets of this form have been excavated, they were used in other prehistoric periods and even the ethnographic past in various areas of North Africa (Garve et al., 2017). It is possible that dealers are taking advantage of the cachet attached to the label "Saharan Neolithic". Some of these exotic and fragile items might be modern fakes produced for the collectors' market.

\section{Fishing weights}

Several sellers offered, usually in groups, small somewhat roughly made longitudinally perforated elongated biconical and cylindrical fired clay objects $40-60 \mathrm{~mm}$ long and some $220 \mathrm{~mm}$ diameter that they sold as Neolithic fishing net weights, adding emotively "from the vanished lakes and rivers of the Green Sahara". These weights seem to have been found in some numbers, but equally could be fakes. Despite their brutalist form, it seems that they were marketed as suitable for making ethnic jewellery (with an interesting and evocative story).

\section{Neolithic beads and pendants}

The collecting of historical beads is a relatively popular hobby in Northern America and Europe, and African beads (for example African trade beads) are an important part of the material available (Francis 1994; Simak etal., 20I0). Fifteen sellers listed in Table I (some of whom specialised in collectable beads and jewellery) had 717 loose and nine 
strings of Saharan Neolithic beads and pendants on sale (with an expected retail value of 5690 dollars). These were sold as wearable antiquities. The forms of these beads was variable, but the most common were rather roughly-made discoid or doughnut-shaped examples apparently of white or transparent quartz, discoid beads of varying size of ostrich eggshell, and small cylindrical beads of carnelian. There were also some small cylindrical beads of Amazonite, but since their findspots re not known they have nothing to add to our knowledge of the routes by which such beads (Zerboni et al., 20I8) were distributed. Alongside the beads were also a number of larger discoidal or block-shaped pieces of a variety of stones sold as Neolithic pendants (or "amulets").

The authenticity of attribution of these items is unclear, we still know very little about the characteristics of "grounded" (excavated) assemblages of Neolithic and later beads from different parts of this large region (cf. Bar-Yosef 2013). ${ }^{4}$ Among the beads shown in the sellers' photos are examples that can be paralleled in excavated assemblages of later beads (such as from the region of Sudan in Meroitic times). Some beads can be seen to have perforations that must have been made with a modern metal drill. Ostrich eggshell beads also have a long history in the region (Bednarik 20II). It may well be that not all of them come from looting of Neolithic sites, but an unknown number of these beads might be more "primitive" looking ones salvaged from the breakingdown of recent bead strings containing old beads of mixed ages and merely marketed as Saharan Neolithic.

\section{Nile Valley}

Some of the early pre-dynastic cultures of the Nile valley overlap with the Neolithic Green Sahara period, while the middle pre-dynastic period probably correlates with its end. Several of the hollow base point types among the flint material on the US market related to the pre-dynastic and early dynastic cultures of the Nile Valley. Greenwell (2005: 36) notes the parallels and adds: "these types do not appear to be terribly rare but, due to the Egyptian antiquities laws, are difficult to find, except in old collections". There is indeed relatively little from this region on eBay. Some material from Egypt apparently from such an old collection was being sold by one US dealer who is offering 95 Neolithic blades and knives from the shoreline of Birket Qarun in the Fayum (with findspots recorded) found in the 1930s and 40s from a "private collector from Port Townsend Washington". He also has 2I Acheulean handaxes from several sites in Egypt and Libya. Another seller has I8 pieces of ancient (?) knapping waste made of Libyan desert glass that were "found many years ago on an eroded site in Egypt". Apart from these, there are four other antiquities (of the Naqada culture), reputedly from old collections, being sold by an additional three US dealers.

4 I would like to thank Dr Lenka Varadzinová for discussing beads with me. 
$330 \mid$ Discussions and criticism

\section{Later Objects}

Although outside the scope of this presentation, to put the sales offers of Saharan Neolithic material in context, it may be noted that the same online sellers generally have relatively few items dating to the 6800 year span after the end of the Saharan Final Neolithic. This material clearly does not have such wide appeal as the prehistoric "Green Sahara" artefacts. A few of the sellers of Table i have Berber, Tuareg, Moorish and Bedouin ethnographic items (jewellery, decorative objects, knives). ${ }^{5}$ A number also have ethnographic items (masks, sculptures, jewellery etc.) from all over Africa and "tribal art" from other regions too. There are quite a number of glass and stone beads from Northern Africa on eBay.com in general (including by these sellers) that are labelled Roman, and also some that are sold as "Islamic". Not all of these however seem ancient. In contrast to the rest of northern Africa, antiquities from Ancient Egypt have long been eagerly collected. As a result, at the time of the 2019 search, there were 742I artefacts and pseudo-artefacts dated to the period from the Old Kingdom to Ptolemaic times on ebay.com.

\section{Prices}

The prices established by the sellers for Buy-it-now sales were tabulated. The prices for Oldowan tools was between 13-270 US dollars, while Acheulean handaxes ranged in price from 30 to $180 \$$ (with most falling between 40 and 90), though one US dealer priced his from 90 to 200 dollars (in both cases were outliers up to $400 \$$ ). Aterian points were mostly priced in the range $13-23 \$$ each. Though there were more expensive ones, Neolithic polished axes tended to range in price between 20 and 150 dollars, with the majority falling in the range 30-70 dollars. Most of the larger and heavier tool types (grinders, pounders, quern fragments) were priced relatively highly, reflecting their scarcity on the market. Beads were variously-priced, but often expensive compared to the other artefacts (some for 50-60 dollars for an individual item). Projectile points however were sold comparatively cheaply. While a few could be priced 30, 50 or 60 dollars each, the bulk of them (97\%) fell into the price range I-I8 dollars. Among these, $8 \mathrm{I} \%$ of the whole sample were priced $\mathrm{I}-7 \$$, and the majority of these $(26, \mathrm{I} 5$ and $\mathrm{I} 5 \%$ of the whole sample respectively) were in the range $\mathrm{I}-3 \$$. Though there is obviously money to be made from this trade, if the bulk of these points are selling at such a price, artefact hunters themselves are not getting much of a share of it unless they bring huge numbers of artefacts to the middlemen (see Brodie 1998).

5 The total number being offered on eBay.com by these and other sellers at the time of the survey was something like 853 . 


\section{Sales Dynamics}

The overall numbers of items on sale at the time of the survey is shocking. In total 6I sellers were offering for sale 8817 artefacts and beads with a total value assessed at over $\$ 70,000$. Not all of these items would have been sold at once (indeed a few of the objects seen in January 2020 were the same ones that had been offered in August the previous year), but in many cases when they were, they would be replaced with others. ${ }^{6}$ On eBay, articles that were sold through bidding and started with a low starting price very frequently attracted some bidding and were sold within a few days of being put online. Unless the dealer employed a "shill" to push bids up (illegal, but known to happen on eBay), slow bidding means that an item may sell for lower sums (this is the attraction of auctions of course for the buyer). Many buyers therefore prefer to place the items online for a higher buy-it-now price, which tends to postpone the sale, which may have to be relisted several times.

As noted, eBay is constructed in such a way that it is difficult to follow the activities of individuals. The majority of the feedback records on past transactions are often set up (by the seller) to hide some of the details of the transactions, and therefore cannot provide a picture of the volume and rate of turnover. Analysing these records is also very time consuming. For this overview, the past sales of only a sample of the dealers that handled relatively high volumes of Saharan Neolithic material were analysed. They fall into two groups, sellers that only retailed items by auction (bidding) and a larger group that listed items that primarily had (higher) buy it now prices. Sellers of the first group consistently managed to sell all or at least $80 \%$ of their periodic sales offers in the past six months. The "Buy-it-now" sellers examined varied in their success rate. In half the cases examined, in six months some of them sold $50 \%$ of their offered stock while others moved $20-25 \%$. The other half however had minimal sales of this type of material (even though buyers were more interested their other objects). A major factor seems to be their pricing policy, many sellers (and in particular those that also specialise in "tribal art") seem to overestimate the worth of the objects they have on offer. It seems therefore that some sellers can sell the equivalent of their entire current offer of Saharan Neolithic material in a year's trading (and continually replace what is online), while others will sell about half of it, while others still will sell considerably less. Fay (2013: 20I-202) found that $52 \%$ of the artefacts (and $74 \%$ of the coins) on eBay were sold during the four months when she was observing it in 2008.

For many of these dealers, Saharan Neolithic artefacts are not the only product they sell, but six months trading in such items alone earned about half the dealers surveyed an income of several hundred dollars, while others were making sums of I500 to 3500 dollars from such sales. It is clearly impossible to generalise from such figures, but it is clear that selling North African lithics can be quite profitable.

6 See footnote 1. 
332 Discussions and criticism

\section{CONFLICT ANTIQUITIES}

Politically, much of northern Africa has been relatively unstable in post-Colonial times and recent events there invite reflection on the relationship of the antiquities trade to social inequality and injustice (Harsch 2006; Krishnan et al., 2016), in particular whether some of these artefacts may even be conflict antiquities (Stevenson 2016; Hardy 2017). The Western Sahara is still a contested region, there has been recent conflict and civil war in Algeria (199I-2002), Libya (20I4-present), Sudan (1983-2005 and 2003-present) and Mali (2012-2019). The 20II Arab Spring revolution was followed by antiquities looting in Egypt and Libya. Unrest and militant action in Niger (2007-2009 and later) also affected the areas where some of these artefacts are coming from. Some of the artefacts now surfacing on the antiquities market thus may well have been the products of earlier looting during the breakdown of law and order and originally sold in order to raise money to support armed conflict (Lehr 2019). In such a situation, as with other antiquities generally, their handling, sale and purchase raise a number of moral and ethical questions.

\section{CONCLUSION}

The number of Saharan Neolithic artefacts currently on sale online is disturbingly high. Although it seems that the turnover of this trade is not as rapid as other artefact types, it is still significant enough to substantiate the claim that since the mid I990s, several million artefacts have been removed from sites in the Sahara and scattered on the international antiquities market, and this process is ongoing. This is looting on an industrial scale. It seems that some areas have been stripped of diagnostic material. As long as a lucrative market for this material exists, the extent of the areas stripped out will only spread.

In the Sahara, the majority of Neolithic sites consist of shallow surface spreads of material and the bases of negative features exposed by deflation in the desert conditions that have prevailed here for six millennia. As such, they are extremely fragile and sensitive to interference. A single unrecorded search episode removing the most collectible (and therefore diagnostic) material will irreversibly alter not only the composition of the site assemblage but, above all, the pattern of distribution of material that constitutes the main body of evidence that the site holds. In analysing prehistoric landscapes in the desert, the stratigraphic, artefactual and environmental data from single sites are not the main type of evidence. Desert surveys analyse the cumulative distribution of sites and off-site findspots not only in relation to each other but also to the geological effects of changing landscapes and natural environment. Rendering unrecognizable sites and findspots forming part of those patterns disrupts that research. 
Much of the current discussion or collection-driven exploitation of the archaeological record ("archaeological looting") is predicated on equating archaeological technique and research solely with excavation and, therefore is based on the notion of "diggers", individuals that excavate holes into archaeological stratigraphy to remove buried artefacts, like the haqueros discussed by Atwood (2004) or metal detectorists. In the UK, the removal by the latter of material that is "only" taken from the disturbed topsoil is taken as an exoneration of this collection-driven activity (Deckers et al., 2018: 323). Paradoxically, the recording and analysis of the patterns of material in that same topsoil is a basic technique of landscape archaeology (a discipline that largely developed in Britain).

The damage caused by collection-driven stripping of surface sites does not manifest itself in a visibly legible form as a site honeycombed with holes that can produce shocking photographs at ground level and the progress of which across a site can be tracked on time-lapse aerial and satellite photos (as seen recently in looting in Iraq and Syria, and in looting on the limes in Bulgaria). Yet, although so rarely highlighted, this types of damage (seen elsewhere too, e.g., in Victoria in Australia: Lever 2016) is devastating to the archaeological record.

The collection-driven destruction of the Sahara's Stone Age archaeological resources threatens to obliterate part of the world's cultural heritage and also profoundly affects our ability to understand the human past of the region. About $15 \%$ of the world's population today live in arid regions. These and other areas are being placed under increasing stress as we face the prospect of imminent climate change. In such circumstances, attempting to understand better both how human activity may affect environmental change in this fragile ecosystem (Wright 20I7), but also how human communities adapted to those changes is potentially of more than mere academic interest (Brooks 20I3).

Fifteen years ago, Brodie and Renfrew (2005) looked at the impact of collectiondriven exploitation on the world's archaeological heritage, and came to the conclusion that the response of archaeologists and opinion-makers to the issue was, as they put it, inadequate. Since then, nothing much has changed, the damage has gone on apace. Artefact hunting, alongside erosion and other geological processes, agricultural expansion, military activity, road construction, vandalism or mining and other extractive industries, is just one of the threats to archaeological sites in the fragile environment of the Sahara region. Yet it is one that arguably we can still be doing something about. Why aren't we? 
334

Discussions and criticism

\section{REFERENCES}

Atwood, R. 2004. Stealing history: tomb raiders, smugglers, and the looting of the ancient world. New York.

Bar-Yosef, D. 2013. Towards a typology of stone beads in the Neolithic Levant. Journal of Field Archaeology 38(2): I29-I42.

Barker, A. 20I8. Looting, the antiquities trade, and competing valuations of the past. Annual Review of Anthropology 47: 455-474.

Bednarik, R. G. 20II. About ostrich eggshell beads. The Bead Forum 59: 2-8 (accessible online: http:// www.ifrao.com/wp-content/uploads/2015/02/97Ostrich.pdf).

Berner, J. 2002. American Indian artifacts: genuine or reproduction? Roswell.

Brodie, N. 1998. Pity the poor middlemen. Culture Without Context 3: 7-9.

Brodie, N. 2015. The internet market in antiquities. In F. Desmarais (ed.), Countering Illicit Traffic in cultural goods: the global challenge of protecting the World's Heritage, II-2O. Paris.

Brodie, N. 2017. Virtually gone! The internet market in antiquities. In Proceedings of the 6th International Conference of Experts on the Return of Cultural Property; Overseas Korean Cultural Heritage Foundation: Seoul, Korea, 2017, 190-204. Seoul.

Brodie, N. and Renfrew, C. 2005. Looting and the world's archaeological heritage: the inadequate response. Annual Review of Anthropology 34: 343-36r.

Brodie, N. and Tubb, K. W. 2002. Illicit antiquities: the theft of culture and the extinction of archaeology. London.

Brooks, N. 2013. Beyond collapse: climate change and causality during the Middle Holocene Climatic Transition, 6400-5000 years before present. Geografisk Tidsskrift - Danish Journal of Geography II2(2): 93-IO4.

Bruhns, K. 2000. www.plunderedpast.com. Society for American Archaeology Bulletin I8(2): 14-15.

Chippindale, C. and Gill, D. 20or. On-line auctions: a new venue for the antiquities market. Culture Without Context 9: 4-I2.

Corbey, R. 2000. Tribal art traffic a chronicle of taste, trade and desire in Colonial and Post-colonial Times. Amsterdam.

Deckers, P., Dobat, A., Ferguson, N., Heeren, S., Lewis, M. and Thomas, S. 20I8. The Complexities of metal detecting policy and practice: a response to Samuel Hardy. Open Archaeology 4(I). Electronic document: https://www.degruyter.com/view/journals/opar/4/1/article-p322.xml.

Di Lernia, S. 2005. Incoming tourism, outgoing culture: tourism, development and cultural heritage in the Libyan Sahara. Journal of North African Studies Io(3-4): 44I-457.

Ede, C. 1976. Collecting Antiquities an Introductory Guide. London.

Fay, E. 20Ir. Virtual artifacts: eBay, antiquities, and authenticity. Journal of Contemporary Criminal Justice 27: 449-464.

Fay, E. 2013. Trading in antiquities on eBay: the changing face of the illicit trade in antiquities. $\mathrm{PhD}$ Thesis, Keele University October 20I3. Electronic document: http://eprints.keele.ac.uk/197/1/Fay $\% 20 \mathrm{PhD} \% 202013$.pdf.

Francis, P. 1994. Beads of the world: a collector's guide with price reference. Atglen.

Garve, R., Garve, M., Türp, J. C. and Meyer, C. G. 20I7. Labrets in Africa and Amazonia: medical implications and cultural determinants. Tropical Medicine and International Health 22(2): 232-240.

Gilmer, M. [2009]. About NWA meteorites. Galactic stone and ironworks. Webpage: http://galactic-stone. com/about-nwa-meteorites/ 
Graburn, N. H. H. 1977. Ethnic and tourist arts: cultural expressions from the Fourth World. Berkeley.

Greenwell, D. 2005. Flint artifacts of North Africa. Self published [no place given].

Hardy, S. A. 2017. Curbing the spoils of war. UNESCO Courier 2017(3): 12-16.

Harsch, E. 2006. Combating inequality in Africa. Africa Renewal 20(2): 16-2I.

Hothem, L. 1992. Fake chipped artifacts: some considerations. Lancaster-Ohio.

Hugot, H.-J. I991. Essai sur les armatures de pointes de flèches du Sahara. Calvisson.

Jury, L. 2005. Art market scandal: British Museum expert highlights growing problem of fake antiquities. Independent, 24 May 2005. Available online: https://www.independent.co.uk/news/uk/this-britain/artmarket-scandal-british-museum-expert-highlights-growing-problem-of-fake-antiquities-222955.html.

Keats, J. 20I4. Playing the meteorite market. Discover magazine, June 20I4: 34. Available online: https:// www.discovermagazine.com/the-sciences/playing-the-meteorite-market).

Keenan, J. 2003. Tourism, development and conservation: a Saharan perspective. In D. J. Mattingly, S. McLaren, E. Savage, Y. al-Fasatwi, and K. Gadgood (eds), Natural resources and cultural heritage of the Libyan Desert: proceedings of a conference held in Libya. I4-2I December 2002, I4-2I. London. Electronic document: http://www.psi.org.uk/ehb/docs/keenan-tourism-200302.pdf.

Keenan, J. 2005. Looting the Sahara: The material, intellectual and social implications of the destruction of cultural heritage (briefing). Journal of North African Studies Io(3-4): 47I-489.

Keenan, J. 2013. The material, intellectual and social implications of the destruction of cultural heritage (Briefing). In J. Keenan (ed.), The Sahara: past, present and future, 214-32. Abingdon.

Klenker, E. K., Dreikluft, R., Milburn, M. and Jiang, Z. 2016. Sahara: material culture of early communities: prehistoric artifacts. Self published [no place given].

Krishnan, N., Ibarra, G. L., Narayan, A., Tiwari, S. and Vishwanath, T. 20I6. Uneven odds, unequal outcomes: inequality of opportunity in the Middle East and North Africa. Washington, D. C., World Bank Group Report. Electronic document: https://bit.ly/2BBjJj8.

Lehr, D. 2019. Art and antiquities: conduits for money laundering and terrorist financing. AML Challenges, Dec. 20I8-Feb. 2019 (Association of Certified Anti-Money Laundering Specialists). Available online: https:// www.acamstoday.org/art-and-antiquities-conduits-for-money-laundering-and-terrorist-financing/.

Lever, M. 20I6. A damaged past: the effects of historical artefact collecting on the stone artefact record. Victoria Excavations, Surveys and Heritage Management in Victoria 5: 47-50.

Lhote, H. 1958. A la Découverte des Fresques du Tassili. Paris.

Lidington, H. 2002. The role of the Internet in removing the "shackles of the saleroom": anytime, anyplace, anything, anywhere. Public Archaeology 2: 67-84.

Mackenzie, S. and Yates, D. 20I6. What is grey about the "grey market" in antiquities. In J. Beckert and M. Dewey (eds), The architecture of illegal markets: towards an economic sociology of illegality in the economy, 70-86. Oxford.

McIntosh, R. J., Togola, T. and McIntosh, S. K. 1995. The Good Collector and the premise of Mutual respect Among Nations. African Arts 28: 60-69.

Sawaged, T. 1999. The Collecting Culture: an exploration of the collector mentality and archaeology's response. Nebraska Anthropologist I2I: 80-88.

Simak, E., Dreibelbis, C., Liechty, J., Donato, M. and Dubin, L. S. 2010. African beads: Jewels of a continent. Denver.

Stanish, C. 2009. Forging ahead. Or, how I learned to stop worrying and love eBay. Archaeology 62(3): 58-60. 
336 Discussions and criticism

Stevenson, A. 20I6. Conflict antiquities and conflicted antiquities: addressing commercial sales of legally excavated artefacts. Antiquity 90(349): 229-236.

Tokely, J. 2006. Rescuing the Past: The cultural heritage crusade. Exeter-Charlottesville.

UNESCO 1970. Convention on the means of prohibiting and preventing the illicit import, Export and transfer of ownership of cultural property. Paris.

van Ham, T., Leukfeldt, R., Bremmers, B., Stol, W. and van Wijk, A. 20II. The art of the Internet: a study of illegal online trading in cultural goods. The Hague.

Vernet, R. and Le Quellec, J.-L. 20I7. Recension de: Eckhard Klenkler, Robert Dreikluft, Mark Milburn and Z. Jiang 20I6. Sahara. Material culture of early communities. Les Cahiers de l' l'Association des Amis de l'Art Rupestre Saharien I9: 295-297.

Vitelli, K. D. 2000. E-commerce in antiquities. Society for American Archaeology Bulletin 18(4): 4-5.

Whittaker, J. C. and Stafford, M. 1999. Replicas, fakes, and art: the twentieth century Stone Age and its effects on archaeology. American Antiquity 64(2): 203-2I4.

Wright, D. K. 20I7. Humans as agents in the termination of the African Humid Period Front. Earth Science 5: I-I4.

Zerboni, A., Salvatori, S., Vignola, P. and Mohammed, A. A. 20I8. The long-distance exchange of amazonite and increasing social complexity in the Sudanese Neolithic. Antiquity 92(365): II95-I209. 